\title{
Gemcitabine, Dexamethasone, and Cisplatin (GDP) versus Dexamethasone, Cytarabine, and Cisplatin (DHAP) as Salvage Chemotherapy for Patients With Relapsed or Refractory Diffuse Large B-cell Lymphoma (DLBCL)
}

\author{
Soheir S. Ismaeil, Tarek Hussein K., Mohamed Yassin M. and Nawal Eltohamy M. \\ Department of Clinical Oncology and Nuclear Medicine, Faculty of Medicine, Ain Shams University
}

Purpose: To study the efficacy and toxicity of dexamethasone, cisplatin, and gemcitabine in comparison to dexamethasone, cisplatin, and cytarabine in treating patients with first relapse or refractory diffuse large B-cell lymphoma (DLBCL).

Patients and methods: sixty two patients with histological diagnosis of relapsed or refractory DLBCL were included, 43 males and 19 females, with a median age 48 years (range 19-63). Twenty-eight (45.2\%) patients had stage IV at relapse, 17 (27.4\%) stage II, 10 (16.1\%) stage III, and 7 (11.3\%) stage I. Treatment consisted of two arms (each arm consisted of 31 patients): ARM I "cisplatin $75 \mathrm{mg} / \mathrm{m} 2$ on day 1, dexamethasone $40 \mathrm{mg}$ on days $1-4$, and gemcitabine $1000 \mathrm{mg} / \mathrm{m} 2$ on days 1 and 8 , given every 21 days" and ARM II, Patients received "cisplatin $75 \mathrm{mg} / \mathrm{m} 2$ IV over 24 hours on day 1, dexamethasone as in arm A, and cytarabine $2 \mathrm{gm} / \mathrm{m} 2$ IV over 3 hours every 12 hours on day 2, given every 21 days". A total of 330 chemotherapy cycles were administered, with a mean of 5.4 cycles per patient (range 2-6).

Results: Among the sixty two eligible patients treated, there were three early deaths, including one patient in group A and two patient in group B. Four patients in group A and three patients in group B had disease progression during treatment. The overall response rate was $65 \%(29 \% \mathrm{CR}$ rate, $38 \% \mathrm{PR}$ rate $)$ in arm $\mathrm{A}$ and $67.6 \%(32.2 \% \mathrm{CR}$ rate, 35.4\% $\mathrm{PR}$ rate) in arm $\mathrm{B}$. The median disease free survival time was 10 months, 9 months (95\% CI, 6.078 to 11.922 months), the median Progression free survival time was 4 months (95\% CI, 2.108 to 5.892 months), 4 months (95\% CI, 3.028 to 4.972 months), the median overall survival time was 20 months (95\% CI, 14.377 to 25.623 months), 21 months (95\% CI, 9.352 to 32.648 months) for arm A\& B, respectively.

Hematologic toxicities were comparable between the two arms with greater number of patients developed grade III, IV neutropenia in arm B. The most common grade III or IV hematological toxicities were neutropenia and thrombocytopenia, neutopenia was $62.9 \%$ in group A and $70 \%$ in group B, febrile neutopenia was significantly higher in group B (53.8\%) compared to (22.2\%) in group A. Grade III or IV thrombocytopenia was observed in $29.7 \%$ among both groups. Other hematological toxicities are comparable in both groups. Stomatitis and infection were higher in arm B. Stomatitis was $36 \%, 69.2 \%$, Infection was $24 \%, 42.3 \%$ for arm A\& B, respectively.

Conclusion: The study revealed no significant differences between GDP and DHAP as regarding response rate, OS, DFS and PFS in treatment of relapsed or refractory DLBCL. Toxicity was higher in DHAP group.

Key words: gemcitabine, dexamethasone, GAP, cytarabine, (DHAP).

Corresponding Author: Dr. Mohamed yassin M. $\quad$ E-mail: doctoryasso@gmail.com

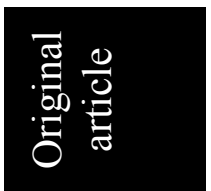

\section{INTRODUCTION}

Non Hodgkin lymphomas (NHLs) are the most commonly occurring hematologic malignancies in the United States. They represent $4 \%$ to $5 \%$ of all new cancer cases. There is 65,540 men and women $(35,380$ men and 30,160 women) diagnosed with and 20,210 men and women died of NHL in $2010^{1}$.

DLBCLis themostcommon type of NHLapproximately $30 \%$ of all lymphoid malignancies. It is associated with an aggressive natural history, with a median survival of less than one year in untreated patients ${ }^{2}$.
Although the adoption of R.CHOP as the new standard of care has improved outcomes for DLBCL, patients still relapse. The standard approach for fit patients with DLBCL has been to proceed toward salvage therapy and consolidation with Autologous Stem Cell Trasplantation "ASCT "3.

Several standard regimens exist for salvage lymphoma therapy including ICE (ifosphamide, carboplatin, etoposide), ESHAP (etoposide, methyl prednisolone, high-dose cytarabine, cisplatin), DHAP (dexamethasone, 
cisplatin, cytarabine), and GDP (dexamethasone, cisplatin, gemcitabine) with various response rates. The choice of salvage therapy is still debated ${ }^{2}$.

This is a phase III prospective clinical trial conducted in Ain Shams University Hospitals comparing the efficacy and toxicities of GDP and DHAP as salvage chemotherapy in first relapse or refractory DLBCL.

\section{PATIENTS AND METHODS}

\section{Patient eligibility:}

Histologically confirmed DLBCL patients in first relapse or refractory after first line chemotherapy (CHOP or CHOP+rituximab) were eligible for this study. Other eligibility criteria included the presence of clinically or radiologically documented disease at least one bidimensionally measurable lesion; at least 16 years old but not more than 65 years, Eastern Cooperative Oncology Group performance status (ECOG-PS) $\leq 2$; adequate bone marrow function (absolute neutrophil count $\geq 1.5 \times 103 / \mathrm{ml}$, platelet count $\geq 100 \times 103 / \mathrm{ml}$, hemoglobin $\geq 10 \mathrm{~g} / \mathrm{dL}) ;$ adequate renal and hepatic function (serum creatinine $\leq 1.5 \mathrm{mg} / \mathrm{dl}$, hepatic enzymes $\leq$ $2.5 \times$ upper normal limit, bilirubin $\leq 1.5$ times upper limit of normal). Exclusion criteria were active infections or cardiovascular disease or other severe comorbid diseases that could have interfered with the trial. Written informed consent was obtained from all patients. The trial was approved by the Ethics and Scientific Committees at Faculty of Medicine, Ain Shams University.

\section{Treatment protocol:}

From December 2010 to December 2012, a total of 62 patients were enrolled in this trial with a median follow up of 18 months (range 7-24 months).

Arm A consisted of 31 patients received cisplatin $75 \mathrm{mg} / \mathrm{m}^{2}$ IV over 60 minutes on day 1 , dexamethasone $40 \mathrm{mg}$ IV on days $1-4$, and gemcitabine $1000 \mathrm{mg} / \mathrm{m}^{2} \mathrm{IV}$ over 30 minutes on days 1 and 8 , treatment was given every 21 days. While Arm B also consisted of 31 patients, received cisplatin $75 \mathrm{mg} / \mathrm{m}^{2}$ IV over 24 hours on day 1 , dexamethasone as in arm A, and cytarabine $2 \mathrm{gm} / \mathrm{m}^{2} \mathrm{IV}$ over 3 hours every 12 hours on day 2, treatment was given every 21 days. Antiemetic premedication included 5-HT3 antagonists, full supportive therapy were given as needed. All patients were scheduled to receive 6 cycles of therapy if there was no evidence of disease progression or severe toxicity.

\section{Evaluation of response and toxicity:}

Pretreatment evaluation included, complete history stressing upon B symptoms, the physical examination; ECOG-PS (4); chest and abdominal computerized tomography scan (CT scan), neck C.T. scan in case of neck disease; complete blood count, kidney and liver function tests, $\mathrm{LDH}$ and bone marrow trephine biopsy. Tumor response was measured using a CT scan (that defined the initial extent of the disease) every two cycles and at the end of treatment. Bone marrow biopsy was repeated at the end of treatment for those patients with lymphomatous infiltration on entry into the trial. Response was evaluated in accordance to the International Workshop NHL response criteria ${ }^{5}$. Toxicity was recorded every 2 cycles according to the Common Toxicity Criteria of the National Cancer Institute (NCICTC, Version 2.0) ${ }^{6}$.

\section{Statistical analysis:}

The Kaplan-Meier Survival Analysis, Log rank test, Student T test, Chi Square test and Fisher's exact test were used to determine the statistical analysis in this study.

\section{RESULTS}

\section{Patient's characteristics:}

Patients' and tumor characteristics at baseline are listed in (Table 1).

\section{Response and survival:}

Among the sixty two eligible patients treated, with a median follow up of 18 months (range 7-24 months), there were three early deaths, including one patient in group A and two patient in group B. Four patients in group A and three patients in group B had disease progression during treatment. After completing 6 cycles, there were $9 \mathrm{CR}$ $(36 \%)$ and 14 PR (48\%) in group A, while in group B were $10 \mathrm{CR}(38.5 \%)$ and $11 \mathrm{PR}(42.3 \%)$, but a $65 \%$ response rate $(29 \% \mathrm{CR}$ rate, $38 \% \mathrm{PR}$ rate) in group A and $67.6 \%$ response rate $(32.2 \% \mathrm{CR}$ rate, $35.4 \% \mathrm{PR}$ rate) in group $\mathrm{B}$ were observed if all patients are considered (Table 2).

Among patients in Arm A, the median Overall Survival (OS) time was 20 months (95\% CI, 14.37725.623 months), the survival rate for this group was $56.7 \%$ at 18 month and $39 \%$ at 24 months (Table 3). The median disease free survival time was 10 months (Table 4). The median Progression free survival time was 4 months (95\% CI, 2.108 to 5.892 months). While, Arm B, the median OS time was 21 months $(95 \% \mathrm{CI}$, $9.066-12.934$ months). The survival rate for this group was $54.8 \%$ at 18 month and $44 \%$ at 24 months. The median disease free survival time was 9 months $(95 \%$ CI, 6.078 to 11.922 months). The median Progression free survival time was 4 months (95\% CI, 3.028 to 4.972 months) (Table 5).

\section{Toxicity:}

Toxicity assessed during treatment every 2 cycles. A total of 55 patients were evaluable for toxicity 27 patients in group A and 28 patients in group B after removal 
of patients who developed disease progression. One patient died in group A due to febrile neutropenia, and two patients in group B, one due to febrile neutropenia and the other due to thrombocytopenia. One patient in group A was removed from the trial due to severe thrombocytopenia and neutropenia.

The most common hematological toxicities were Grade III or IV neutropenia and thrombocytopenia, neutopenia was $62.9 \%$ in group A and $70 \%$ in group B, but febrile neutopenia was significantly higher in group $\mathrm{B}$, occurred in 14 patients $(53.8 \%)$ compared to 6 patients $(22.2 \%)$ in group A. Grade III or IV thrombocytopenia was observed in $29.7 \%$ among both groups. Other hematological toxicities were comparable in both groups.

In the current trial, there was no significant difference in percentage of non hematological toxicity among both groups of patients except in stomatitis and infection which were higher in group B. In group A 9 patients (36\%) developed stomatitis compared to 18 patients $(69.2 \%)$ in group B. Infection occurred in 6 patients (24\%) among group A and in 11 patients (42.3) among group B, was ranging between grade I, II and III (12\% grade I, $8 \%$ grade II, 4\% grade III) in group A and was ranging between grade I, II and III (23\% grade I, 3.8\% grade II, $15.3 \%$ grade III) in group B (Table 6).

In total, 330 cycles were administered, with a mean of 5.4 cycles per patient (range 2-6). Of all the planned infusions, there were 57 dose modifications $(17.2 \%$ of cycles), all of them were candidate for chemotherapy dose modification due to encountered toxicities and continued their planned therapy. The most common cause of dose modification was neutropenia, thrombocytopenia and renal toxicity.

Table 1: Description and comparison of Patient characteristics and Prognostic factors between GDP and DHAP treatment groups

\begin{tabular}{|c|c|c|c|c|c|c|c|c|c|}
\hline & & \multicolumn{2}{|c|}{ GDP } & \multicolumn{2}{|c|}{ DHAP } & \multicolumn{2}{|c|}{ Total } & \multirow{2}{*}{$P$} & \multirow{2}{*}{ Sig. } \\
\hline & & $N$ & $\%$ & $N$ & $\%$ & $N$ & $\%$ & & \\
\hline \multirow{2}{*}{ Age group } & $=<50$ Year & 12 & $38.7 \%$ & 16 & $51.6 \%$ & 28 & $45.2 \%$ & \multirow{2}{*}{.307} & \multirow{2}{*}{ NS } \\
\hline & $>50$ Years & 19 & $61.3 \%$ & 15 & $48.4 \%$ & 34 & $54.8 \%$ & & \\
\hline \multirow{2}{*}{ Age } & Mean \pm SD & $53.3 \pm 10.6$ & $45.8 \pm 13.23$ & $48.1 \pm 12.1$ & \multirow{2}{*}{.142} & \multirow{2}{*}{\multicolumn{2}{|c|}{ NS }} & & \\
\hline & Range & $25-63$ & $19-63$ & $19-63$ & & & & & \\
\hline \multirow{2}{*}{ Sex } & Male & 21 & $67.7 \%$ & 22 & $71.0 \%$ & 43 & $69.4 \%$ & \multirow{2}{*}{.783} & \multirow{2}{*}{ NS } \\
\hline & Female & 10 & $32.3 \%$ & 9 & $29.0 \%$ & 19 & $30.6 \%$ & & \\
\hline \multirow{3}{*}{ Performance status } & 0 & 5 & $16.1 \%$ & 5 & $16.1 \%$ & 10 & $16.1 \%$ & \multirow{3}{*}{1.00} & \multirow{3}{*}{ NS } \\
\hline & 1 & 20 & $64.5 \%$ & 20 & $64.5 \%$ & 40 & $64.5 \%$ & & \\
\hline & 2 & 6 & $19.4 \%$ & 6 & $19.4 \%$ & 12 & $19.4 \%$ & & \\
\hline \multirow{2}{*}{ Extra nodal } & No & 25 & $80.6 \%$ & 20 & $64.5 \%$ & 45 & $72.6 \%$ & \multirow{2}{*}{.155} & \multirow{2}{*}{ NS } \\
\hline & Yes & 6 & $19.4 \%$ & 11 & $35.5 \%$ & 17 & $27.4 \%$ & & \\
\hline \multirow{4}{*}{ Stage at presentation } & Stage 1 & 5 & $16.1 \%$ & 7 & $22.6 \%$ & 12 & $19.4 \%$ & \multirow{4}{*}{.585} & \multirow{4}{*}{ NS } \\
\hline & Stage 2 & 15 & $48.4 \%$ & 14 & $45.2 \%$ & 29 & $46.8 \%$ & & \\
\hline & Stage 3 & 5 & $16.1 \%$ & 2 & $6.5 \%$ & 7 & $11.3 \%$ & & \\
\hline & Stage 4 & 6 & $19.4 \%$ & 8 & $25.8 \%$ & 14 & $22.6 \%$ & & \\
\hline \multirow{4}{*}{ Stage at relapse } & Stage 1 & 4 & $12.9 \%$ & 3 & $9.7 \%$ & 7 & $11.3 \%$ & \multirow{4}{*}{.743} & \multirow{4}{*}{ NS } \\
\hline & Stage 2 & 10 & $32.3 \%$ & 7 & $22.6 \%$ & 17 & $27.4 \%$ & & \\
\hline & Stage 3 & 5 & $16.1 \%$ & 5 & $16.1 \%$ & 10 & $16.1 \%$ & & \\
\hline & Stage 4 & 12 & $38.7 \%$ & 16 & $51.6 \%$ & 28 & $45.2 \%$ & & \\
\hline B symntoms & Negative & 22 & $71.0 \%$ & 19 & $61.3 \%$ & 41 & $66.1 \%$ & 421 & NS \\
\hline B symptoms & Positive & 9 & $29.0 \%$ & 12 & $38.7 \%$ & 21 & $33.9 \%$ & .421 & NS \\
\hline & Normal & 19 & $61.3 \%$ & 16 & $51.6 \%$ & 35 & $56.5 \%$ & 442 & NS \\
\hline LDH & Elevated & 12 & $38.7 \%$ & 15 & $48.4 \%$ & 27 & $43.5 \%$ & .442 & NS \\
\hline & 0 & 8 & $25.8 \%$ & 7 & $22.6 \%$ & 15 & $24.2 \%$ & & \\
\hline & 1 & 11 & $35.5 \%$ & 9 & $29.0 \%$ & 20 & $32.3 \%$ & & \\
\hline IPI & 2 & 8 & $25.8 \%$ & 10 & $32.3 \%$ & 18 & $29.0 \%$ & .896 & NS \\
\hline & 3 & 4 & $12.9 \%$ & 5 & $16.1 \%$ & 9 & $14.5 \%$ & & \\
\hline & CHOP & 23 & $74.2 \%$ & 25 & $80.6 \%$ & 48 & $77.4 \%$ & 544 & NS \\
\hline Previous chemotherapy & RCHOP & 8 & $25.8 \%$ & 6 & $19.4 \%$ & 14 & $22.6 \%$ & .544 & $\mathrm{NS}$ \\
\hline Response to previous & $\begin{array}{l}\text { Complete } \\
\text { response }\end{array}$ & 14 & $45.2 \%$ & 16 & $51.6 \%$ & 30 & $48.4 \%$ & .611 & NS \\
\hline & Refractory & 17 & $54.8 \%$ & 15 & $48.4 \%$ & 32 & $51.6 \%$ & & \\
\hline & $<3$ Months & 4 & $28.6 \%$ & 2 & $12.5 \%$ & 6 & $20.0 \%$ & & \\
\hline Time to disease & 3-12 Months & 8 & $57.1 \%$ & 12 & $75.0 \%$ & 20 & $66.7 \%$ & .512 & NS \\
\hline & $>12$ Months & 2 & $14.3 \%$ & 2 & $12.5 \%$ & 4 & $13.3 \%$ & & \\
\hline
\end{tabular}


Table 2: Description and comparison between GDP and DHAP treatment groups

\begin{tabular}{|c|c|c|c|c|c|c|c|c|c|}
\hline & & \multicolumn{6}{|c|}{ Group } & \multirow{3}{*}{$P$} & \multirow{3}{*}{ Sig } \\
\hline & & \multicolumn{2}{|c|}{ GDP } & \multicolumn{2}{|c|}{ DHAP } & \multicolumn{2}{|c|}{ Total } & & \\
\hline & & $\mathbf{N}$ & $\%$ & $\mathbf{N}$ & $\%$ & $\mathbf{N}$ & $\%$ & & \\
\hline \multirow{4}{*}{ Response after 6 cycles } & Complete response & 9 & $36.0 \%$ & 10 & $38.5 \%$ & 19 & $37.3 \%$ & \multirow{4}{*}{0.783} & \multirow{4}{*}{ NS } \\
\hline & Partial response & 12 & $48.0 \%$ & 11 & $42.3 \%$ & 23 & $45.1 \%$ & & \\
\hline & Stationary & 4 & $16.0 \%$ & 4 & $15.4 \%$ & 8 & $15.7 \%$ & & \\
\hline & Progression & 0 & $0.0 \%$ & 1 & $3.8 \%$ & 1 & $2.0 \%$ & & \\
\hline
\end{tabular}

Table 3: Description and comparison of overall survival between GDP and DHAP treatment groups

\begin{tabular}{|c|c|c|c|c|c|c|c|c|c|}
\hline \multirow{2}{*}{$\begin{array}{l}\text { Treatment modality } \\
\text { GDP }\end{array}$} & \multirow{2}{*}{$\begin{array}{l}\mathbf{N} \\
25\end{array}$} & \multirow{2}{*}{$\begin{array}{c}\text { Mean } \\
18.879\end{array}$} & \multicolumn{2}{|c|}{ 95\% Confidence Interval } & \multirow{2}{*}{$\begin{array}{l}\text { Median } \\
20.000\end{array}$} & \multicolumn{2}{|c|}{$\begin{array}{l}\text { 95\% Confidence } \\
\text { Interval }\end{array}$} & \multirow[t]{2}{*}{$P^{*}$} & \multirow[t]{2}{*}{ Sig } \\
\hline & & & 16.462 & 21.296 & & 14.377 & 25.623 & & \\
\hline DHAP & 26 & 18.041 & 15.396 & 20.686 & 21.000 & 9.352 & 32.648 & .737 & NS \\
\hline Overall & 51 & 18.463 & 16.663 & 20.263 & 21.000 & 16.191 & 25.809 & & \\
\hline
\end{tabular}

Table 4: Description and comparison of disease free survival between GDP and DHAP treatment groups

\begin{tabular}{lcccccccc}
\hline Treatment modality & N & Mean & $\mathbf{9 5 \%}$ Confidence Interval & Median & $\mathbf{9 5 \%}$ Confidence Interval & $\boldsymbol{P}^{*}$ & Sig \\
\hline GDP & 9 & 13.875 & 10.300 & 17.450 & 10.000 & & & \\
\hline DHAP & 10 & 11.222 & 7.257 & 15.188 & 9.000 & 6.078 & 11.922 & 0.217 \\
\hline Overall & 19 & 13.163 & 9.989 & 16.337 & 10.000 & 8.029 & 11.971 & \\
\hline
\end{tabular}

Table 5: Description and comparison of progression free survival between GDP and DHAP treatment groups

\begin{tabular}{|c|c|c|c|c|c|c|c|c|c|}
\hline Treatment modality & $\mathbf{N}$ & Mean & $\begin{array}{l}\text { 95\% Confidence } \\
\text { Interval }\end{array}$ & Median & $\begin{array}{l}\text { 95\% Confidence } \\
\text { Interval }\end{array}$ & $P^{*}$ & Sig & & \\
\hline GDP & 16 & 6.000 & 4.175 & 7.825 & 4.000 & 2.108 & 5.892 & \multirow{3}{*}{0.256} & \multirow{3}{*}{ NS } \\
\hline DHAP & 16 & 4.875 & 3.735 & 6.015 & 4.000 & 3.028 & 4.972 & & \\
\hline Overall & 32 & 5.418 & 4.352 & 6.484 & 4.000 & 2.643 & 5.357 & & \\
\hline
\end{tabular}


Kasr-El-Aini Journal Of Clinical Oncology And Nuclear Medicine

Vol. 9 | No. 3-4

2013

Soheir S. Ismaeil et al.

Table 6: Description and comparison of hematologic and non hematological toxicities between GDP and DHAP treatment groups

\begin{tabular}{|c|c|c|c|c|c|c|c|c|}
\hline & & & & & & & & \\
\hline & & & & & & & & $\boldsymbol{P}$ \\
\hline & & $N$ & $\%$ & $N$ & $\%$ & $N$ & $\%$ & \\
\hline Anemia & No & 2 & $7.4 \%$ & 6 & $21.4 \%$ & 8 & $14.5 \%$ & \\
\hline Anemıa & Yes & 25 & $92.2 \%$ & 22 & $78.5 \%$ & 47 & $85.4 \%$ & .872 \\
\hline & 0 & 2 & $7.4 \%$ & 6 & $21.4 \%$ & 8 & $14.5 \%$ & \\
\hline & & 13 & $48.1 \%$ & 14 & $50.0 \%$ & 27 & $49.0 \%$ & \\
\hline Anemia grade & $\frac{1}{2}$ & $\frac{15}{8}$ & $\frac{40.170}{29.6 \%}$ & $\frac{14}{5}$ & $\frac{0.070}{17.9 \%}$ & $\frac{21}{13}$ & $\frac{49.070}{23.6 \%}$ & .033 \\
\hline & 3 & 4 & $14.8 \%$ & 3 & $10.7 \%$ & 7 & $12.7 \%$ & \\
\hline & No & 3 & $11.1 \%$ & $\frac{3}{3}$ & $10.7 \%$ & $\frac{1}{6}$ & $10.9 \%$ & \\
\hline Neutropenia & Yes & 24 & $88.9 \%$ & 25 & $89.3 \%$ & 49 & $89.1 \%$ & .962 \\
\hline & 0 & 3 & $11.1 \%$ & 3 & $10.7 \%$ & 6 & $10.9 \%$ & \\
\hline & 1 & 2 & $7.4 \%$ & 3 & $10.7 \%$ & 5 & $9.1 \%$ & \\
\hline Neutropenia grade & 2 & 5 & $18.5 \%$ & 2 & $7.1 \%$ & 7 & $12.7 \%$ & .454 \\
\hline & 3 & 8 & $29.6 \%$ & 5 & $17.9 \%$ & 13 & $23.6 \%$ & \\
\hline & 4 & 9 & $33.3 \%$ & 15 & $53.6 \%$ & 24 & $43.6 \%$ & \\
\hline & No & 10 & $37.0 \%$ & 16 & $59.3 \%$ & 26 & $48.1 \%$ & \\
\hline Thrombocytopenia & $\frac{1 \mathrm{vo}}{\mathrm{Yes}}$ & $\frac{10}{17}$ & $63.0 \%$ & $\frac{10}{11}$ & $40.7 \%$ & $\frac{20}{28}$ & $\frac{40.170}{51.9 \%}$ & .102 \\
\hline & 0 & 9 & $33.3 \%$ & 16 & $59.3 \%$ & 25 & $46.3 \%$ & \\
\hline & 1 & 8 & $29.6 \%$ & 1 & $3.7 \%$ & 9 & $16.7 \%$ & \\
\hline Thrombeutononis orodo & 2 & 2 & $7.4 \%$ & 1 & $3.7 \%$ & 3 & $5.6 \%$ & \\
\hline Thrombocytopenia grade & 3 & 7 & $25.9 \%$ & 7 & $25.9 \%$ & 14 & $25.9 \%$ & .120 \\
\hline & $\frac{3}{4}$ & $\frac{1}{1}$ & $3.7 \%$ & $\frac{1}{1}$ & $3.7 \%$ & $\frac{17}{2}$ & $3.7 \%$ & \\
\hline & 5 & 0 & $.0 \%$ & 1 & $3.7 \%$ & 1 & $1.9 \%$ & \\
\hline & No & $\frac{v}{21}$ & $77.8 \%$ & $\frac{1}{13}$ & $50.0 \%$ & $\frac{1}{34}$ & $64.2 \%$ & \\
\hline Febrile neutropenia & Yes & $\frac{1}{6}$ & $22.2 \%$ & 14 & $53.8 \%$ & $\frac{79}{19}$ & $35.8 \%$ & .035 \\
\hline & 0 & 21 & $77.8 \%$ & $\frac{17}{13}$ & $50.0 \%$ & 34 & $64.2 \%$ & \\
\hline & 1 & $\frac{21}{0}$ & $.0 \%$ & $\frac{12}{8}$ & $30.8 \%$ & $\frac{7}{8}$ & $15.1 \%$ & \\
\hline & 2 & 0 & $.0 \%$ & 2 & $7.7 \%$ & 2 & $3.8 \%$ & \\
\hline Febrile neutropenia grade & 3 & 4 & $14.8 \%$ & 3 & $11.5 \%$ & $\frac{2}{7}$ & $13.2 \%$ & .003 \\
\hline & 4 & 1 & $3.7 \%$ & 0 & $.0 \%$ & 1 & $1.9 \%$ & \\
\hline & $\frac{7}{5}$ & 1 & $3.7 \%$ & $\frac{0}{1}$ & $3.7 \%$ & $\frac{1}{2}$ & $3.8 \%$ & \\
\hline & No & 5 & $20.0 \%$ & 3 & $11.5 \%$ & 8 & $15.6 \%$ & \\
\hline Nausea & Yes & 20 & $80.0 \%$ & $\frac{5}{23}$ & $88.4 \%$ & $\frac{0}{43}$ & $84.9 \%$ & .343 \\
\hline & 0 & $\frac{20}{6}$ & $24.0 \%$ & $\frac{25}{3}$ & $\frac{0 . .7 \%}{11.5 \%}$ & 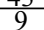 & $17.6 \%$ & \\
\hline & $\frac{0}{1}$ & 10 & $\frac{2.0 \%}{40.0 \%}$ & $\frac{11}{11}$ & $42.3 \%$ & 21 & $\frac{11.070}{41.1 \%}$ & \\
\hline Nausea grade & $\frac{1}{2}$ & $\frac{10}{6}$ & $24.0 \%$ & $\frac{11}{3}$ & $11.5 \%$ & 9 & $17.6 \%$ & .421 \\
\hline & 3 & 3 & $12.0 \%$ & $\frac{5}{6}$ & $23.0 \%$ & 9 & $17.6 \%$ & \\
\hline & 4 & 0 & $.0 \%$ & 3 & $11.5 \%$ & 3 & $5.8 \%$ & \\
\hline & No & 11 & $44.0 \%$ & $\frac{11}{11}$ & $42.3 \%$ & 22 & $43.1 \%$ & \\
\hline Vomiting & Yes & 14 & $56.0 \%$ & $\frac{11}{15}$ & $57.6 \%$ & $\frac{22}{29}$ & $56.8 \%$ & .865 \\
\hline & 0 & $\frac{17}{11}$ & $44.0 \%$ & $\frac{15}{11}$ & $42.3 \%$ & $\frac{25}{22}$ & $43.1 \%$ & \\
\hline & 1 & $\frac{11}{6}$ & $\frac{4.070}{24.0 \%}$ & $\frac{11}{5}$ & $\frac{42.0 \%}{19.2 \%}$ & $\frac{22}{11}$ & $\frac{45.170}{21.5 \%}$ & \\
\hline Vomiting grade & $\frac{1}{2}$ & 4 & $16.0 \%$ & 5 & $19.2 \%$ & $\frac{11}{9}$ & $17.6 \%$ & .872 \\
\hline & 3 & 4 & $16.0 \%$ & $\frac{2}{4}$ & $15.3 \%$ & 8 & $15.6 \%$ & \\
\hline & $\frac{3}{4}$ & 0 & $.0 \%$ & 1 & $3.8 \%$ & $\frac{0}{1}$ & $1.9 \%$ & \\
\hline Ctometitig $0 \mathrm{C}$ & No & 16 & $64.0 \%$ & 8 & $30.8 \%$ & 24 & $47.1 \%$ & 017 \\
\hline Stomatitis & Yes & 9 & $36.0 \%$ & 18 & $69.2 \%$ & 27 & $52.9 \%$ & .017 \\
\hline & 0 & 16 & $64.0 \%$ & 8 & $30.8 \%$ & 24 & $47.1 \%$ & \\
\hline Stomotitis arod & 1 & $\frac{10}{5}$ & $20.0 \%$ & 9 & $34.6 \%$ & 14 & $27.5 \%$ & \\
\hline Stomatitis grade & $\frac{1}{2}$ & $\frac{\pi}{4}$ & $16.0 \%$ & $\frac{5}{6}$ & $\frac{5.00}{23.1 \%}$ & $\frac{14}{10}$ & $19.6 \%$ & .066 \\
\hline & 3 & 0 & $.0 \%$ & 3 & $11.5 \%$ & 3 & $5.9 \%$ & \\
\hline & No & 16 & $64.0 \%$ & 14 & $53.8 \%$ & 30 & $58.8 \%$ & \\
\hline Diarrhea & $\frac{1 \mathrm{vo}}{\text { Yes }}$ & $\frac{10}{9}$ & $36.0 \%$ & $\frac{17}{12}$ & $46.2 \%$ & 21 & $41.2 \%$ & .461 \\
\hline & 0 & 16 & $64.0 \%$ & 12 & $46.2 \%$ & $\frac{1}{28}$ & $54.9 \%$ & \\
\hline & $\frac{0}{1}$ & $\frac{10}{5}$ & $20.0 \%$ & $\frac{12}{6}$ & $23.1 \%$ & 11 & $21.6 \%$ & \\
\hline Diarrhea grade & 2 & 4 & $16.0 \%$ & 4 & $15.4 \%$ & 8 & $15.7 \%$ & .200 \\
\hline & 3 & 0 & $.0 \%$ & $\frac{7}{4}$ & $15.4 \%$ & $\frac{0}{4}$ & $7.8 \%$ & \\
\hline 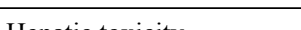 & No & $\frac{0}{15}$ & $60.0 \%$ & 17 & $65.4 \%$ & 32 & $62.7 \%$ & 100 \\
\hline Hepatic toxicity & Yes & 10 & $40.0 \%$ & 9 & $34.6 \%$ & 19 & $37.2 \%$ & .493 \\
\hline & 0 & 15 & $60.0 \%$ & 17 & $65.4 \%$ & 32 & $62.7 \%$ & \\
\hline Hepatic toxicity grade & 1 & 10 & $40.0 \%$ & 8 & $30.7 \%$ & 18 & $35.2 \%$ & .452 \\
\hline & $\frac{1}{2}$ & 0 & $8.0 \%$ & 1 & $3.8 \%$ & 1 & $1.9 \%$ & \\
\hline 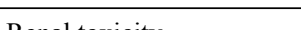 & No & 19 & $76.0 \%$ & $\frac{1}{17}$ & $65.4 \%$ & $\frac{1}{36}$ & $70.6 \%$ & 106 \\
\hline Renal toxicity & Yes & 6 & $24.0 \%$ & $\frac{x}{9}$ & $\frac{0.7 \%}{34.6 \%}$ & $\frac{\pi}{15}$ & $29.4 \%$ & .406 \\
\hline & 0 & 19 & $76.0 \%$ & 17 & $65.4 \%$ & 36 & $70.6 \%$ & \\
\hline Renal toxicity grade & 1 & $\frac{17}{5}$ & $20.0 \%$ & $\frac{17}{5}$ & $\frac{0.470}{19.2 \%}$ & $\frac{50}{10}$ & $19.6 \%$ & .088 \\
\hline & 2 & 1 & $4.0 \%$ & 4 & $15.4 \%$ & 5 & $9.8 \%$ & \\
\hline & No & 19 & $76.0 \%$ & $\frac{7}{15}$ & $57.6 \%$ & 34 & $66.6 \%$ & \\
\hline Infection & Yes & 6 & $24.0 \%$ & $\frac{11}{11}$ & $42.3 \%$ & 17 & $33.3 \%$ & .040 \\
\hline & 0 & $\frac{0}{19}$ & $\frac{24.0 \%}{76.0 \%}$ & $\frac{11}{15}$ & $\frac{42.070}{57.6 \%}$ & $\frac{11}{34}$ & $66.6 \%$ & \\
\hline Infection orode & 1 & 3 & $12.0 \%$ & 6 & $23.0 \%$ & 9 & $17.6 \%$ & \\
\hline Infection grade & $\frac{1}{2}$ & $\frac{3}{2}$ & $8.0 \%$ & $\frac{0}{1}$ & $3.8 \%$ & 3 & $5.7 \%$ & .048 \\
\hline & $\frac{2}{3}$ & $\frac{2}{1}$ & $\frac{0.070}{4.0 \%}$ & $\frac{1}{4}$ & $\frac{5.070}{153 \%}$ & $\frac{3}{5}$ & $\frac{5.170}{9.8 \%}$ & \\
\hline & No & 4 & $16.0 \%$ & 7 & $26.9 \%$ & 11 & $12.5 \%$ & 050 \\
\hline Fatigue & Yes & 21 & $84.0 \%$ & $\frac{1}{19}$ & $73.1 \%$ & $\frac{11}{40}$ & $78.4 \%$ & .059 \\
\hline & 0 & $\frac{1}{4}$ & $16.0 \%$ & $\frac{17}{7}$ & $26.9 \%$ & 11 & $12.5 \%$ & \\
\hline$\Gamma$ tion & 1 & $\frac{1}{11}$ & $44.0 \%$ & $\frac{1}{6}$ & $23.1 \%$ & 17 & $33.3 \%$ & 207 \\
\hline Fatigue grade & $\frac{1}{2}$ & $\frac{11}{10}$ & $40.0 \%$ & 10 & $38.5 \%$ & $\frac{11}{20}$ & $37.7 \%$ & .297 \\
\hline & 3 & 0 & $.0 \%$ & 3 & $11.5 \%$ & $\frac{2}{3}$ & $5.7 \%$ & \\
\hline & No & 15 & & $\frac{16}{16}$ & & 27 & & \\
\hline Neurosensory & Yes & 10 & $40.0 \%$ & 10 & $38.5 \%$ & 24 & $47.1 \%$ & .210 \\
\hline & 0 & 15 & $60.0 \%$ & 16 & $61.5 \%$ & 27 & $52.9 \%$ & \\
\hline Neurosensory grade & $\frac{0}{1}$ & $\frac{13}{7}$ & $\frac{00.070}{28.0 \%}$ & $\frac{10}{5}$ & $\frac{01.5 \%}{19.2 \%}$ & $\frac{21}{12}$ & $\frac{32.970}{23.5 \%}$ & 446 \\
\hline & 2 & 3 & $12.0 \%$ & 5 & $19.2 \%$ & 12 & $23.5 \%$ & \\
\hline & No & $\frac{78}{18}$ & $72.0 \%$ & 18 & $69.2 \%$ & 36 & & \\
\hline Ototoxicity & Yes & $\frac{10}{7}$ & $28.0 \%$ & $\frac{10}{8}$ & $30.8 \%$ & $\frac{5}{15}$ & $29.4 \%$ & .828 \\
\hline & 0 & $\frac{1}{18}$ & $72.0 \%$ & $\frac{0}{18}$ & $69.2 \%$ & $\frac{15}{36}$ & $70.6 \%$ & \\
\hline Ototoxicity grade & $\frac{0}{1}$ & $\frac{10}{6}$ & $\frac{12.0 \%}{24.0 \%}$ & $\frac{10}{7}$ & $\frac{07.260}{26.9 \%}$ & $\frac{30}{2}$ & $\frac{10.070}{3.9 \%}$ & .972 \\
\hline & $\frac{1}{2}$ & 1 & $4.0 \%$ & 1 & $3.8 \%$ & 13 & $25.5 \%$ & \\
\hline
\end{tabular}




\section{DISCUSSION}

The choice of salvage therapy is still debated. Results of a clinical trial indicate that the combination chemotherapy consisting of gemcitabine, cisplatin, and dexamethasone (GDP) produced responses in approximately half $(49 \%)$ of patients with recurrent or refractory NHL. It concluded that GDP is an active regimen in B-cell NHL and can be administered with acceptable toxicity ${ }^{7}$. Because DHAP has been widely used all over the world and was the salvage regimen of the PARMA study, it was used here as comparator.

The results of DHAP group in the current trial were slightly higher than the results achieved in a phase II study conducted by Velasquez and colleagues in 1988 , in which 90 patients with relapsing or refractory lymphoma were treated with DHAP regimen. Of the 83 evaluable patients, $31 \%$ of the patients achieved complete response and $26.5 \%$ of the patients achieved partial response while $19 \%$ of the patients had progressive disease. The 2 year OS was $25 \%$.

The higher results could be attributed to the better clinico-pathological characteristics of the patients recruited in our study. The patients in Velasquez trial had previous exposure to an alkylating agent and Adriamycin in diverse combination regimens including "second and third generation regimens." Furthermore, 58 patients (64\%) had also received VP-16 and methotrexate as part of inductive therapy or as a salvage trial. In addition, six patients had failed a cisplatin-based regimen (compared to patients in our trial received $\mathrm{CHOP} \pm$ rituximab), 72 patients only had intermediate grade lymphoma, while all patients in the current trial had diffuse large cell lymphoma, 52 patients (57\%) did not achieve CR with primary induction therapy (refractory patients) comparing to $48.4 \%$ in our trial. Extranodal disease was rather frequent $86 \%$ compared to $35.5 \%$ in our trial ${ }^{8}$.

Comparing GDP arm in the current trial to Crump`s trial, in which 50 patients with recurrent or refractory DLBCL or its variants received GDP. After 2 cycles, there were 8 complete responses CR; $16 \%$ (13.3\% in the current trial) and 17 partial responses PR; 33\% (53.3\% in the current trial), there was an overall response rate (RR) of $49 \%$, the RR after completion of all protocol chemotherapy was $53 \%$ (65\% in our trial), the median time to progression from the start of GDP was 3.1 months $(95 \% \mathrm{CI}=2.3-9.2)$. The CR rate and the median time to progression were comparable to our current study matched rate ${ }^{7}$.

Comparison of ICE versus DHAP was done by Abali and his colleagues on 53 patients, Response could be evaluated in 49 patients ( $36 \mathrm{NHL}$ and $13 \mathrm{HD}$ ).
ICE seems to have higher rates of response than DHAP regimen as in DHAP group 11 (22.5\%) achieved CR and 17 (35\%) achieved PR, leading to an overall response rate (ORR: $\mathrm{CR}+\mathrm{PR}$ ) of $57.5 \%$. In the evaluable ICE group $(\mathrm{n}=22)$ rates of $\mathrm{CR}, \mathrm{PR}$, and ORR were $27 \%$, $41 \%$ and $68 \%$ and in the DHAP group $(\mathrm{n}=27)$ rates of CR, PR, and ORR were $18 \%, 30 \%$ and $48 \%(P=0.24$, for $\mathrm{ORR})^{9}$.

In the Collaborative Trial in Relapsed Aggressive Lymphoma (CORAL) study, a phase III multicenter randomized trial designed to compare the efficacy of R-ICE and R-DHAP in patients with previously treated DLBCL followed by ASCT, The median age of the 396 patients enrolled (R-ICE, $\mathrm{n}=202$; R-DHAP, $\mathrm{n}=194$ ) was 55 years. Similar response rates were observed after three cycles of R-ICE $(63.5 \%$; $95 \% \mathrm{CI}, 56 \%$ to $70 \%$ ) and R-DHAP (62.8\%; $95 \mathrm{CI}, 55 \%$ to $69 \%$ ). There was no significant difference between R-ICE and R-DHAP for 3-year event-free survival (EFS) or overall survival ${ }^{10}$.

Our current study results regarding comparing the response and the survival between the two treatment modalities are similar to CORAL study results (the larger trial) but unlike Abali and his colleague`s trial.

Factors affecting response rates in CORAL study were refractory disease/relapse less than versus more than 12 months after diagnosis $(46 \% \mathrm{v} 88 \%$, respectively), IPI of more than 1 versus 0 to $1(52 \% \mathrm{~V}$ $71 \%$, respectively), and prior rituximab treatment versus no prior rituximab $(51 \% \mathrm{v} 83 \%$, respectively). Threeyear EFS was affected by prior rituximab treatment versus no rituximab ( $21 \%$ v $47 \%$, respectively), relapse less than versus more than 12 months after diagnosis $(20 \% \mathrm{v} 45 \%$, respectively), and IPI of 2 to 3 versus 0 to $1(18 \% \text { v } 40 \% \text {, respectively })^{10}$.

These factors affecting response rates in CORAL study were similar with our data, as a better response was observed in patients with lower IPI and CR after the previous chemotherapy. But the previous rituximab did not have a significant effect on our study's response in both groups separately. These may be attributed to our low number of patients with previous mabthera $8(25.8 \%)$ in GDP and $6(19.4 \%)$ in DHAP groups.

Our current study as regarding GDP toxicity was quite comparable to Crump and his colleague's trial. Grade III and IV neutropenia occurred in $63 \%$ of patients (was $62.9 \%$ in ours) and $14 \%$ experienced an episode of febrile neutropenia (was $22.2 \%$ in ours). Grade III and IV thrombocytopenia occurred in $28 \%$ of patients (was $29.7 \%$ in ours) ${ }^{8}$. 
In Velasquez trial neutropenia grade IV was $48 \%$ compared to $53.6 \%$ in DHAP group in our trial, but the current study reported a lower incidence of grade IV thrombocytopenia $7.4 \%$ versus $35.6 \%$ in Velasquez trial that could be explained by better clinico-pathological characteristics of the patients in the current trial as all of them had DLBCL and were at first relapse ${ }^{8}$.

\section{CONCLUSION}

In conclusion, the study had found no significant differences between GDP and DHAP as regarding response rate, OS, DFS and PFS in treatment of relapsed or refractory DLBCL. DHAP was more toxic as regarding febrile neutopenia, stomatitis and infection. Also the current results matched the published results of phase II trials that used GDP and DHAP as salvage chemotherapy.

\section{REFERENCES}

1. Howlader N, Noone AM, Krapcho M, Neyman N, Aminou R, et al. (2011): SEER Cancer Statistics Review, 1975-2008, National Cancer Institute. Bethesda, MD, http://seer.cancer.gov/csr/1975_2008/, based on November 2010 SEER data submission, posted to the SEER web site, 2011

2. Flowers CR, Sinha R, Vose JM. Improving Outcomes for Patients with Diffuse Large B-Cell Lymphoma. Ca-a Cancer Journal for Clinicians. 2010;60(6):393-408.

3. Sud R, Friedberg JW. Salvage therapy for relapsed or refractory diffuse large B-cell lymphoma: Impact of prior rituximab. Haematologica-the Hematology Journal. 2008;93(12):1776-80.

4. Oken MM, Creech RH, Tormey DC, Horton J, Davis TE,
McFadden ET, et al. Toxicity and response criteria of the Eastern-Cooperative-Oncology-GROUP. American Journal of Clinical Oncology-Cancer Clinical Trials. 1982;5(6):649-55.

5. Cheson BD, Horning SJ, Coiffier B, Shipp MA, Fisher RI, Connors JM, et al. Report of an international workshop to standardize response criteria for nonHodgkin's lymphomas. Journal of Clinical Oncology. 1999;17(4):1244-53.

6. Cancer Therapy Evaluation Program, Common Terminology Criteria for adverse Events, Version 3.0, DCTD, NCI, NIH, DHHS. March 31, 2003(http://cteb. cancer.gov.).

7. Crump M, Baetz T, Couban S, Belch A, Marcellus D, Howson-Jan $\mathrm{K}$, et al. Gemcitabine, dexamethasone, and cisplatin in patients with recurrent or refractory aggressive histology B-cell non-Hodgkin lymphoma - A phase II study by the National Cancer Institute of Canada Clinical Trials Group (NCIC-CTG). Cancer. 2004;101(8):1835-42.

8. Velasquez WS, Cabanillas F, Salvador P, McLaughlin P, Fridrik M, Tucker S, et al. Effective salvage therapy for lymphoma with cisplatin in combination with highdose ARA-C and dexamethasone (DHAP). Blood. 1988;71(1):117-22.

9. Abali H, Ueruen Y, Oeksuezoglu B, Budakoglu B, Yildirim $\mathrm{N}$, Gueler $\mathrm{T}$, et al. Comparison of ICE (ifosfamide - carboplatin - etoposide) versus DHAP (cytosine arabinoside-cisplatin-dexamethasone) as salvage chemotherapy in patients with relapsed or refractory lymphoma. Cancer Investigation. 2008;26(4):401-6.

10. Gisselbrecht C, Glass B, Mounier N, Gill DS, Linch DC, Trneny M, et al. Salvage Regimens With Autologous Transplantation for Relapsed Large B-Cell Lymphoma in the Rituximab Era. Journal of Clinical Oncology. 2010;28(27):4184-90. 